\title{
Bicuspid aortic valve repair by complete conversion from "raphe'd" (type 1) to "symmetric" (type 0) morphology
}

\author{
Thomas G. Gleason, MD
}

Objective: An anterior cusp with a median raphe and false commissure is the most common bicuspid aortic valve phenotype. The cusp is typically restricted and its annular attachment malpositioned, often resulting in significant aortic regurgitation. A novel valve repair strategy was designed to create a symmetric valve to improve both function and durability.

\begin{abstract}
Methods: The technique involves converting the valve into a "symmetric" bicuspid valve by resection of the median raphe, with shortening of the anterior cusp's leading edge; resection of the false commissure with complete anterior annular detachment; enlargement, reinforcement, and translocation of the anterior cusp deeper into the left ventricular outflow tract; and valve-sparing aortic root replacement with concomitant annuloplasty.

Results: A total of 32 patients with a bicuspid valve, aortic insufficiency, and aortic dilatation have undergone valve repair with root replacement. No operative deaths or strokes occurred, and no permanent pacemakers were required. Thirteen patients underwent complete conversion from "raphe'd" to "symmetric" morphology; the others underwent less complex repairs without translocation. The postoperative transvalvular gradients remained normal. Freedom from grade $>1+$ aortic regurgitation and valve-related reoperation was $100 \%$. All 13 symmetric conversion patients have continued to have no or trace aortic regurgitation (grade $<1+$ ) at a mean follow-up of 2.9 years (range, 0.4-7).
\end{abstract}

Conclusions: Repair of the common, "raphe'd" bicuspid phenotype by reconfiguration into "symmetric" morphology is both feasible and desirable, regardless of the degree of preoperative aortic regurgitation. This repair strategy appears durable at the mid-term without evolving signs of deterioration or stenosis, offering a potentially superior option over prosthetic root replacement for younger patients. (J Thorac Cardiovasc Surg 2014;148:2862-8)

Supplemental material is available online.

The morphology of the congenital bicuspid aortic valve (BAV) varies; however, the most common phenotype $(>75 \% \text { incidence })^{1-3}$ is that with a relatively normal noncoronary cusp and a pathologic anterior cusp with both a median raphe and a false commissure (type 1$)^{3}$ The median raphe and false commissure often become markedly sclerotic, resulting in restriction and retraction of the cusp (Figure E1). Many factors can contribute to valvular incompetency, including (1) elongation of the anterior cusp's leading edge relative to the posterior cusp,

From the Department of Cardiothoracic Surgery, Center for Thoracic Aortic Disease, University of Pittsburgh Medical Center, Pittsburgh, Pa.

Disclosures: Authors have nothing to disclose with regard to commercial support.

Received for publication March 24, 2014; revisions received April 20, 2014; accepted for publication May 9, 2014; available ahead of print June 13, 2014.

Address for reprints: Thomas G. Gleason, MD, Department of Cardiothoracic Surgery, Center for Thoracic Aortic Disease, University of Pittsburgh Medical Center, 5200 Centre Ave, Suite 715, Pittsburgh, PA 15232 (E-mail: gleasontg@upmc.edu). $0022-5223 / \$ 36.00$

Copyright (C) 2014 by The American Association for Thoracic Surgery http://dx.doi.org/10.1016/j.jtcvs.2014.05.021 causing prolapse; (2) foreshortening and, thus, restriction of the anterior cusp height (the distance [or height] from the nadir of the basilar attachment to the leading edge at the nodule of Arantius, Figure E2); (3) an inappropriate contour of the anterior annulus (the raphe'd cusp's basilar attachment) in conjunction with the nonfunctional false commissure, causing additional restriction; and (4) annular ectasia, causing flattening or effacement of the subcommissural triangles and making them more obtuse.

The incidence of "symmetric" (type 0) BAV morphology with no raphe and only 2 commissures has been $<10 \%$ among patients with a $\mathrm{BAV}^{3}$ A high percentage of symmetric BAVs will have well-functioning cusps (overall incidence unknown) but can be insufficient owing to aortic root dilatation and annular ectasia. Fenestrations of the leading edge near the commissures have been common in these circumstances. A type 0 BAV will more commonly be competent than a raphe'd (type 1) BAV at aortic root replacement and more easily repairable by focusing on the creation of 2 symmetric cusps with a symmetric annulus and support apparatus (sinus segments). It is this recognition that symmetry will promote competency that prompted the hypothesis that converting a raphe'd BAV to a symmetric BAV could better facilitate repair and render a durable, competent valve. The novelty of the repair strategy 

Abbreviations and Acronyms
$\mathrm{BAV}=$ bicuspid aortic valve
$\mathrm{LV}=$ left ventricular
LVOT $=$ LV outflow tract
VSRR $=$ valve-sparing root replacement

described in the present report is the concept of correcting the cusp, annular, sinus segment, and sinotubular junction (STJ) morphology to create symmetry throughout the root complex (Figure 1). There are 4 critical steps to converting the raphe'd BAV to a symmetric BAV: (1) median raphe resection; (2) cusp detachment with resection of the false commissure; (3) cusp enlargement and translocation into the left ventricular (LV) outflow tract (LVOT); and (4) valve-sparing root replacement (VSRR) with concomitant annuloplasty.

\section{METHODS}

The specific indication for the presented operation has been the presence of type 1 BAV (with an anterior median raphe) and significant aortic insufficiency, with concomitant aortic root dilatation. The operation is tedious and time consuming; thus, patients should be carefully selected based primarily on their excellent overall health. Some LV dilatation can be permitted, provided the LV function is relatively preserved. Patients without any aortic dilatation have not, to date, been considered for this extensive repair. Instead, such patients have been treated with isolated aortic valve repair or replacement. Similarly, patients with an aortic aneurysm but without significant aortic insufficiency or a different (not type 1) morphology have not been considered. These patients have undergone isolated valve-sparing aortic root replacement with or without more conventional valve repair techniques. The evaluation should begin with preoperative echocardiography and computed tomographic angiography to determine the geometry of the native aortic annulus, sinus segments, and STJ. The presented aortic valve repair technique has always been performed in conjunction with VSRR using a modified reimplantation technique $^{4,5}$ and ascending aortic and hemiarch replacement to completely treat the associated ascending aortopathy of the BAV syndrome. All the patients provided informed consent, and the data reviewed were a subset from an institutional review board-approved study.

The operation begins by way of a median sternotomy with conventional cannulation. Myocardial protection is imparted with near-continuous antegrade or retrograde cardioplegia. The patient is cooled, and continuous electrocerebral monitoring is used to direct a safe period of hypothermic circulatory arrest. Retrograde cerebral perfusion was used in all cases.

The ascending aorta was transected, the aortic root was dissected away from the right and main pulmonary arteries, and coronary arterial buttons were created. The entire aortic annulus was dissected to a level $\geq 2 \mathrm{~mm}$ below the annular level circumferentially. Care should be taken to dissect down all the way to the muscle ridge and well below the false (left/right) commissure (and native ventriculoaortic junction). A similar maneuver should be used below the right/noncoronary commissure to, but not through, the membranous septum. The left or noncoronary commissure can be easily dissected to a level well below the nadirs of the annulus. Thus, there is circumferential external dissection of the native annulus that will ultimately allow for complete envelopment by a tailored graft to stabilize the annulus indefinitely.

The sinus segments should be resected, leaving only a 1- to 2-mm cuff of the aorta associated with the annulus (a more aggressive technique that has been used by me since 2004 for all VSRR-reimplantations to better facilitate both annuloplasty and annular fixation to the graft; Figure E3). Each of the 2 symmetric commissures should be retracted with sutures to clearly display the valve. The median raphe and false commissure, in conjunction with an inadequately broad surface area of anterior (raphe'd) cusp, will result in restriction and prolapse of this cusp. The posterior (or noncoronary) cusp will typically be quite healthy and might only have an elongated leading edge, which can be easily corrected with plication as a terminal maneuver after anterior cusp correction.

The anterior cusp should then be detached from the annulus from $1 \mathrm{sym}$ metric commissure to the other to completely free its basilar attachment (Figure 2, A, and Figure E4). The optimal leading edge length should usually be determined by the posterior cusp's leading length. The leading edge of the pathologic anterior cusp should be measured to determine the extent of resection at the leading edge that will be done in conjunction with resection of the median raphe and false commissure. The median raphe and false commissure should be completely resected and the transected leading edges apposed with a 7-0 polytetrafluoroethylene (Gore-Tex; W. L. Gore \& Associates, Inc, Flagstaff, Ariz) suture (Figure 2, B, and Figure E5).

The noncoronary cusp should be meticulously measured to tailor a replica of its shape and dimensions from a harvested segment of native pericardium. The leading edge length and perpendicular measurements of the native cusp depth should be performed serially every few millimeters from 1 commissure to the other using a shortened disposable and flexible ruler. These measurements are used to first create a paper replica of the cusp, which will then serve as a template to create the pericardial cusp. The native pericardium should be stripped of all adipose tissue and the glistening surface oriented on the aortic aspect of the LVOT. This pericardial cusp should be prepared fresh, without tanning, and preserved in heparinized saline (Figure E6). The pericardial cusp has a surface area that is nearly double the surface area of the residual anterior cusp.

The tailored pericardial cusp is then placed below the native anterior cusp, and their leading edges are affixed to each other with a running 80 polytetrafluoroethylene (Gore-Tex; W. L. Gore \& Associates, Inc) suture (Figure 3, A). Each arm of suture should then be tied on the adventitial aspect of the aorta at each respective commissure. The basilar aspect of the pericardial cusp is then attached (translocated) to the LVOT at a position symmetric with the nadir of the basilar attachment of the noncoronary (posterior) cusp. This will typically be 6 to $12 \mathrm{~mm}$ lower than the point at which the native anterior cusp had previously attached. This translocation (reattachment) can be facilitated with running 5-0 polypropylene suture (Figure 3, B). This suture line is begun by passing the suture in a horizontal mattress fashion through the muscular septum out of the base of the heart, where it is tied exteriorly, and then each limb is brought back into the LVOT and simply run up to the commissural attachment, which will be contiguous with the native annulus at the final aspect. The residual free edges of the basilar aspect of the native anterior cusp are then sewn to the midportion of the pericardial cusp using running 6-0 polypropylene suture (Figure 3). Multiple quilting sutures should also be placed throughout the portions of overlapping native and pericardial cusps to ensure good apposition of the tissues to prevent blood from pooling in the created pocket. At this point, 2 symmetrically sized and oriented cusps should be present. The remaining reconstruction involves VSRR using a modified reimplantation technique with annuloplasty that has been previously described. ${ }^{4,5}$ A woven graft should be tailored precisely to configure the optimal root geometry to house the now symmetric bicuspid valve. The optimal root geometry should be determined from the now equivalent cusp depth $^{6}$ (height $[h]$ in Figure E2). The graft diameter is calculated as follows: $2 h(+1$ or $2 \mathrm{~mm}){ }^{4}$ The optimal internal annular diameter $(d)$ will be $d=h / 0.7$; thus, the graft is "necked down" proximally to a diameter of $d$ ( +2 or $3 \mathrm{~mm}$ to accommodate the soft tissues of the native annulus) by placing 4 pleats to create 2 sinus segments (Figure 4 and Figure E3). These should be positioned at a distance roughly two fifths and three fifths of the hemicircumference of the graft relative to the 2 symmetric commissures. 


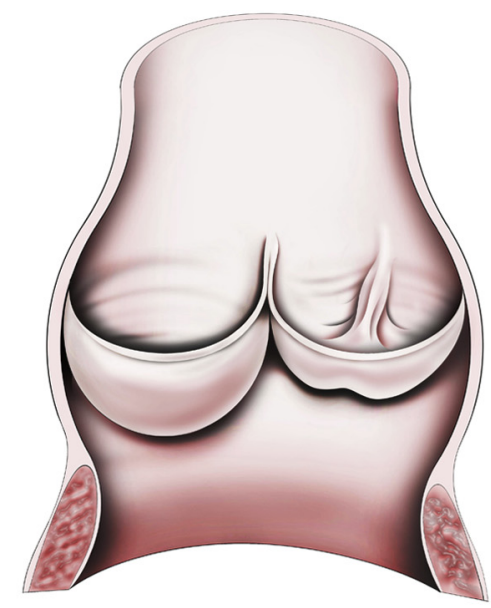

A

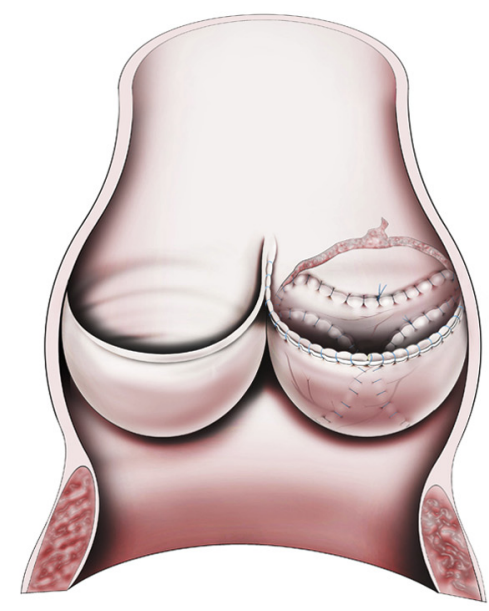

B

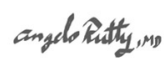

FIGURE 1. A, The appearance of the pathologic "raphe'd" (type 1) bicuspid aortic valve. B, The repaired valve with its complete conversion to a symmetric (type 0) bicuspid aortic valve. Note that the basilar attachment of the enlarged anterior (previously raphe'd) cusp has been translocated to a position lower in the left ventricular outflow tract, symmetric with the noncoronary (posterior) cusp.

The commissural heights are determined, and 2 appropriately sized grooves are cut out of the proximal aspect of the graft to accommodate both the muscle ridge below the previous false commissure and the membranous septum.

The primary annular sutures (2-0 nonpledgeted polyester) are then placed in a horizontal mattress fashion. They should be placed just below the cusp attachment (subannular) point and passed up through the peripheral fibrous or muscular tissues out of the base of the heart. Also, they should strictly follow the coronet shape of the annulus (typically 20-24 sutures will be required; Figure 4). They are then passed through the graft in a coronet-shaped fashion to complete the annuloplasty. It is with this maneuver that the 2 subcommissural triangles can be normalized to correct any annular ectasia. These subcommissural isosceles triangles will often be pathologically obtuse, and the corrected angles should now be acute after performing annuloplasty in this fashion. The tailored graft is placed down to envelop the entire root complex, and the primary annular sutures are tied (Figure 4). The degree of annular reduction will be dictated completely by the calculated optimal internal annular diameter $(d=h / 0.7){ }^{4,5}$ The 2 commissures should be resuspended into the neoroot at a height on the stretched graft that corresponds to the distance from the plane of the nadirs of the annulus to the plane of the peaks of the commissures. The secondary annular suture line should completely affix the annulus to the graft using 2 simple, running sutures, each of which beginning at the nadir of the respective sinus, passing through the annulus, around the 1- to 2-mm cuff of aorta, and through the graft.

The graft is then transected $1 \mathrm{~mm}$ distally to the commissures and a neoSTJ fashioned by pleating the graft at its new distal aspect. Similar to the proximal annular pleats, the STJ pleats should be positioned at two fifths and three fifths of the hemicircumference of the graft relative to the commissures (Figure 4 and Figure E3). The coronary buttons are then transferred. The hemiarch reconstruction can be completed after achieving electrocerebral silence using a second woven graft of a diameter chosen according to the normal distal arch caliber. The patient should then be systemically rewarmed, and attention is drawn back to the aortic root. On completion of the root reconstruction, the arch graft should be trimmed proximally and anastomosed to the STJ of the root graft. Valve performance should be carefully assessed with transesophageal echocardiography after weaning from cardiopulmonary bypass.
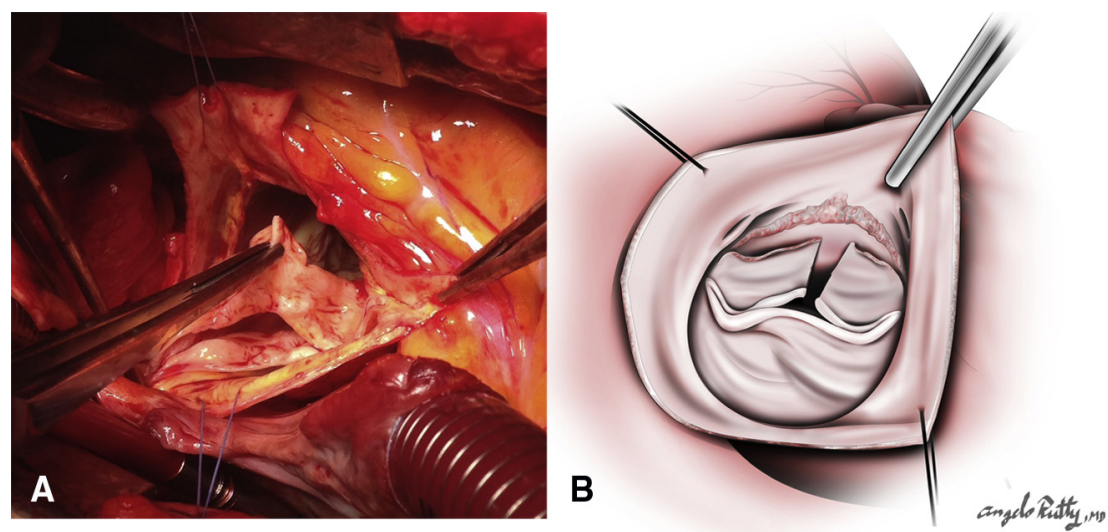

FIGURE 2. A, An intraoperative photograph of a detached, pathologic raphe'd anterior cusp of a bicuspid aortic valve of the most common phenotype. Note the thickened raphe and nodule of Arantius and the clearly demarcated false commissure, which has also been detached. B, Resection of both the median raphe (including the leading edge with the nodule of Arantius) and the false commissure. 

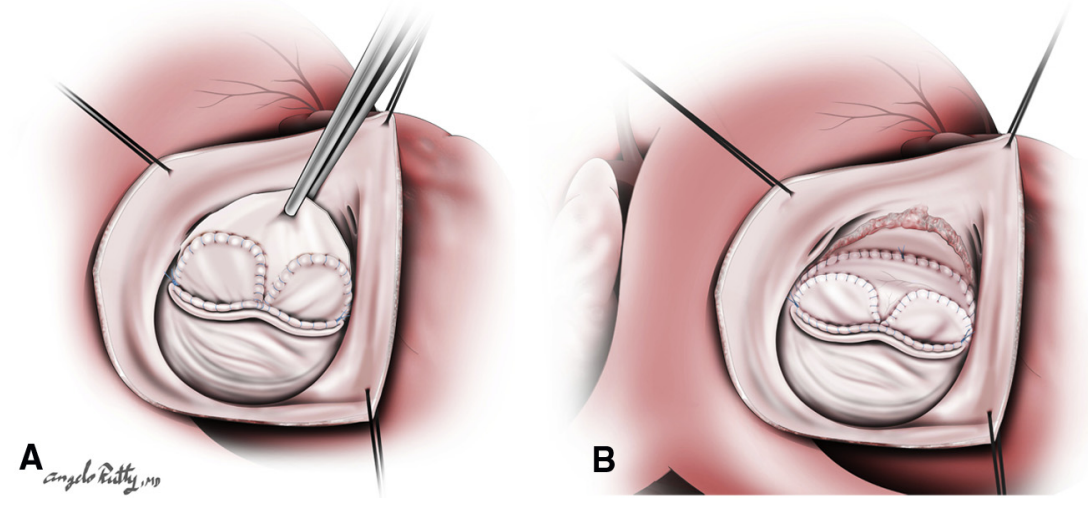

FIGURE 3. A, The reconstructed cusp created by sewing together the native residual anterior cusp and a piece of fresh, native pericardium tailored to a size and shape that is precisely symmetric with the noncoronary (posterior) cusp. Note that the leading edge half of the reconstructed cusp is doubly thick, with the native cusp on top of the fabricated pericardial cusp. B, An illustration of the reconstructed anterior cusp, now sewn back into the left ventricular outflow tract, translocating it to a position well inferior (deeper into the left ventricular outflow tract by 6 to $12 \mathrm{~mm}$ ) from its original annular attachment. It is now symmetrically located relative to the nadir of the noncoronary (posterior) cusp.

All the patients underwent physical examination and echocardiographic surveillance at 1 month postoperatively and annually thereafter. A single computed tomographic angiographic study was performed at 1 year postoperatively.

\section{RESULTS}

A total of 32 patients with BAV, aortic insufficiency, and associated ascending aortic dilatation underwent aortic valve repair, VSRR, and ascending aortic replacement with hemiarch reconstruction from January 2005 to December 2013 (of $>200$ patients who had undergone VSRR and/or aortic valve repair). The symmetric conversion-valve repair technique described was developed in 2007 and applied selectively and concurrently thereafter to 13 patients with type $1 \mathrm{BAV}$ and significant (grade 3-4+) aortic insufficiency who were deemed appropriate. (The other 19 patients underwent more conventional BAV repairs with VSRR that did not include translocation of the reconstructed anterior cusp down into the LVOT.)

The mean patient age was 44 years (range, 22-66) for the entire cohort, with a mean age of 38 years (range, 22-55) for the symmetric conversion group. Most patients were male $(81 \%)$. For those undergoing symmetric conversion, the mean maximal aortic diameter was $51.7 \pm 2.0 \mathrm{~mm}$, the mean LV ejection fraction was 54\% (range, $48 \%-65 \%$ ), and the mean LV end-diastolic diameter was $5.1 \mathrm{~cm}$ (range, 4-6.7). The mean aortic crossclamp, hypothermic circulatory arrest, and cardiopulmonary bypass time was
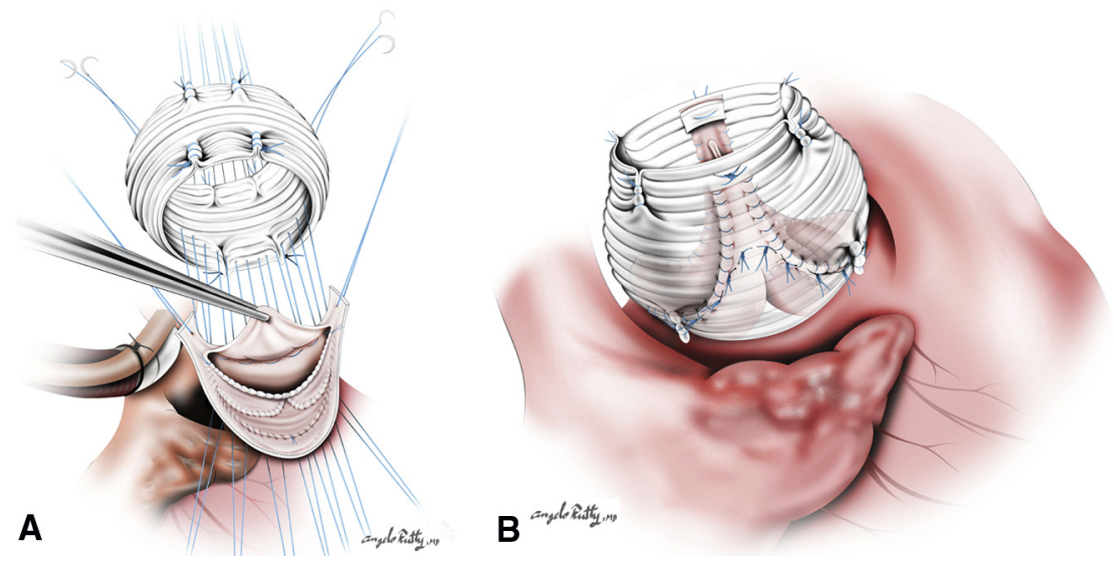

FIGURE 4. After cusp reconstruction and translocation, (A) annuloplasty is facilitated by placing the primary annular sutures through the base of the heart in a subannular position strictly following the coronet shape of the annulus. These sutures are then placed through the tailored polyester graft in a coronetshaped pattern on the graft to reduce the subcommissural angle of the annulus. Note the pleats positioned in the graft to create neosinuses. B, The tailored graft then envelopes the entire root apparatus, illustrating the complete conversion to a "symmetric" bicuspid aortic valve. Note the neosinus creation, the annuloplasty, how the primary suture line's position on the graft will dictate how much more acute the subcommissural isosceles triangle will be, and that the entire subcommissural triangle is supported by the graft material. 
$246 \pm 38,11.5 \pm 0.9$, and $279 \pm 39$ minutes, respectively, for the complete symmetric conversion patients (the crossclamp and cardiopulmonary bypass times were significantly shorter for the remaining cohort).

No hospital deaths or strokes occurred in the entire series. The mean hospital length of stay was 5 days (range, 4-7). No pacemakers were required, and no major complications occurred, neither prolonged ventilation nor renal insufficiency. The mean follow-up period for the entire cohort was 5.3 years (range, 0.4-9.9), and the mean follow-up period for the complete symmetric conversion patients (13 of 32) was 2.9 years (range, 0.4-7). No late deaths or strokes occurred among those who underwent complete symmetric conversion. One late death occurred at 2.5 years in the 19 patients who had not undergone complete symmetric conversion. This patient had developed progressive dilated cardiomyopathy, although the death was noncardiac in nature. The overall long-term survival was 97\% (Figure E7). No valve-related reoperations were required in the entire series.

All patients underwent surveillance echocardiography during follow-up and 1-year postreconstruction computed tomographic angiography (Figure 5). The mean transvalvular gradient for the symmetric conversion patients was 7.2 $\mathrm{mm} \mathrm{Hg}$ (range, 2-12). Freedom from any aortic insufficiency (ie, grade $>0$ or trace) was $100 \%$ for those who underwent complete symmetric conversion. Freedom from aortic insufficiency (grade $>1+$ ) for the remaining patients was $100 \%$. Of the 19 patients in the remaining cohort, 3 had mild (grade 1+) aortic insufficiency at the last followup visit. Only 2 of the complete symmetric conversion patients (aged 22 and 24 years) had significant preoperative LV dilatation (LV end-diastolic diameter, 6.7 and $6.0 \mathrm{~cm}$, respectively), and both had a reduction in the LV enddiastolic diameter to 4.8 and $4.9 \mathrm{~cm}$, respectively, with a LV ejection fraction of $55 \%$ to $60 \%$ for each patient on the last follow-up echocardiogram. The remaining patients have continued to have a normal LV ejection fraction $(55 \%$ $65 \%$ ) with no sign of annular or LV dilatation.

\section{DISCUSSION}

Severe aortic insufficiency affects a large percentage of young and middle-age adults with BAV and its associated ascending aortopathy. Prosthetic aortic valve replacement in this younger population has been suboptimal, rendering either the need for continuous anticoagulation and a lifelong risk of bleeding and thromboembolism with a mechanical valve or expected structural valve deterioration or failure rate of $>35 \%$ after 10 years with a bioprosthetic valve. ${ }^{7}$ Aortic valve repair is a potentially more attractive option for these patients, assuming that it can provide a durability that is significantly better than that currently offered by valve replacement. However, many aortic valve repair strategies have not offered the degree of long-term freedom from reoperation and aortic regurgitation that is ideally

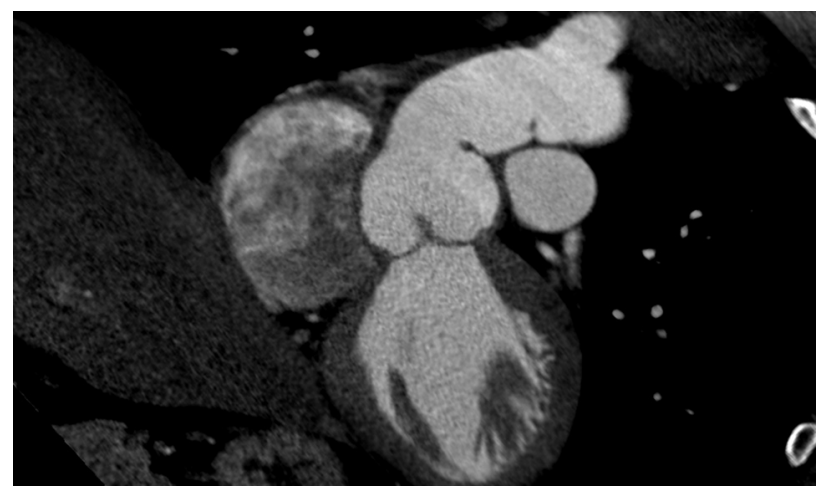

FIGURE 5. A doubly oblique, volume-rendered computed tomographic angiogram after valve repair and valve-sparing root replacement with complete conversion to symmetric anatomy. This valve was converted from a "raphe'd" (type 1) bicuspid aortic valve to a "symmetric" (type 0) bicuspid aortic valve. Note that the 2 cusps are symmetric in size, shape, and position within the left ventricular outflow tract. The neosinus segments are also symmetric in size and shape.

sought. ${ }^{8-17}$ The presented technique was developed with the hope of better long-term durability than other techniques. It was based on the understanding that (1) aortic valve repair of any type will be limited by its ability to minimize stress and strain on the cusps ${ }^{18}$; and (2) it should provide durable structural support throughout the root apparatus to prevent subsequent geometric changes that could result in delayed failure.

The overarching hypothesis was that a raphe'd BAV will be, by its very construct, inadequate and destined to fail over time, largely because of its asymmetric conformation. Without correction of these conformational deficiencies, notably the asymmetry of the cusp sizes, shapes, and annular attachments, valve repair will not be durable. Thus, the design of this repair strategy is to create cusp symmetry and correct the annular ectasia that is typical of the condition. To achieve this goal, the raphe'd BAV is converted to a symmetric BAV by (1) resection of the median raphe with shortening of the anterior cusp's leading edge; (2) resection of the false commissure with complete anterior annular detachment; (3) enlargement, reinforcement, and translocation of the anterior cusp deeper into the LVOT (using native, untanned pericardium); and (4) VSRR with concomitant aortic annuloplasty using a modified reimplantation technique that has been previously described.

The 4 provocative features of the repair strategy are (1) the use of native pericardium to augment the cusp size, (2) the concept of cusp symmetry and translocation, (3) retention of the native leading edge, and (4) the need for annuloplasty. The use of autologous pericardium for valve leaflet (cusp) augmentation or repair began in the 1960s. ${ }^{19,20}$ Since then, discussion has continued about its value, durability, and the optimal method of preparing it for use. In particular, debate has been ongoing about 
whether native pericardium should be fixed (tanned) with glutaraldehyde or used fresh. ${ }^{21}$ The strategy of using fresh pericardium for the augmentation of the anterior cusp described in the present study was based on my longstanding experience working with it in this form, both clinically and in the laboratory. Because a large portion of the native cusp is affixed (sewn) on top of a sheet of tailored pericardium with a large surface area of apposition, there is speculation that the native pericardial cusp will be repopulated with viable stromal cells, which might enhance its durability. To date, we have not seen sclerosis or foreshortening of these fresh pericardial patches used in the manner described. The patient with the longest follow-up (7 years), who had undergone complete symmetric conversion, had native, fresh pericardium used for cusp reconstruction and translocation and has continued to have trace aortic regurgitation with no retraction of the cusp, with good cusp mobility, and a mean transvalvular gradient of 4.7 $\mathrm{mm} \mathrm{Hg}$. Seven other patients have had a follow-up period of 2 to 5 years, also with no signs of retraction or deterioration - these findings are evidence of the potential durability of fresh pericardium used in this context. Aside from a theoretical biologic advantage (using living cells), fresh pericardium, with tissue characteristics very similar to a native cusp, is much easier to work with than tanned or bovine pericardium.

The concept of creating 2 distinctly symmetric cusps was based on the concern that leaving any form of the median raphe or its suspension (the false commissure) would continue to impart restriction of the anterior cusp-the likely source of failure with these valves. Simply creating a symmetric cusp and reattaching it to the native anterior annulus would be inadequate, because it would now impart relative redundancy compared with the noncoronary cusp, unless the anterior cusp's basilar attachment has been moved to a position symmetric with the noncoronary cusp's nadir. Consequently, the cusp attachment should be lowered into the LVOT to a position equal and opposite the nadir of the noncoronary cusp. The entire residual native root and annular structures should be completely enveloped by the tailored graft, which will prevent subsequent annular dilatation (Figure 5). The only portion of the reconstruction not supported by the graft is the muscular septum itself to which the nadir of the reconstructed cusp is attached. However, no evidence, to date, has shown that this portion of the heart (which separates the LVOT and the infundibulum) dilates.

Retention of most of the leading edge of the native raphe'd cusp (except for the central nodule and raphe) serves 2 important purposes. After resection of the raphe and bulky nodule of Arantius, the residual leading edge length is easily matched to the leading edge of the noncoronary cusp simply by joining the 2 cut edges (Figure 3 and Figure E5), given the inherent leading edge redundancy of the raphe'd cusp. Second, retaining most of the native leading edge provides an appropriately thick leading edge surface to promote a more robust coaptation than what a completely pericardial cusp could render.

Finally, the need for concomitant annuloplasty has been previously described, ${ }^{4,5,11}$ and the crux of facilitating an optimal annuloplasty in this setting is the creation of a coronet-shaped subannular primary suture line for the reimplantation technique. ${ }^{5,22}$ By performing this initial graftaffixing maneuver, the geometry of the subcommissural triangles, which are often flattened (becoming more obtuse) because of the ectasia, will be corrected to now appear as a more acute isosceles triangle. This annular correction will impart better cusp coaptation, again with the aim of better durability. Despite passage of primary annular sutures through the graft in a coronet shape, graft material will remain outside the subcommissural triangle to a level below the plane of the nadirs of both sinus segments and will prevent any future annular or subannular ectasia (Figures 4 and 5).

The presented operation is tedious and technically demanding and will need continued refinement to make it quicker and more easily reproducible. Despite the rather lengthy reconstruction times, the operative results have been excellent, without significant morbidity or mortality. However, the patients chosen to date have been highly selected for their good health and relatively preserved LV function. In very favorable circumstances (ie, young adults), it has been applied in a few patients with LV dilatation, but this has been done cautiously. The presentation of this technique was purposely delayed to this point to establish a reasonable follow-up period and local reproducibility. Its durability has now been demonstrated in the present small series to the mid-term, with no aortic insufficiency among those patients who have undergone the complete conversion to symmetric anatomy. The overall value of this strategy will not be adequately measured until a significant number of patients with 10- to 20-year follow-up are available, but these preliminary results are encouraging.

The author thanks Angelo Rutty, MD, for his magnificent illustrations of the techniques outlined in this report, Thomas D. Scollon, III, CST, for his loyalty and technical support in orchestrating these repairs, and Katie L. Scollon, PAC, for her assistance in completing these repairs and caring for these patients.

\section{References}

1. Rinnstrom D, Engstrom KG, Johansson B. Subtypes of bicuspid aortic valves in coarctation of the aorta. Heart Vessels. 2014;29:354-63.

2. Jackson V, Petrini J, Caidahl K, Eriksson MJ, Liska J, Eriksson P, et al. Bicuspid aortic valve leaflet morphology in relation to aortic root morphology: a study of 300 patients undergoing open-heart surgery. Eur J Cardiothorac Surg. 2011;40: e118-24.

3. Sievers HH, Schmidtke C. A classification system for the bicuspid aortic valve from 304 surgical specimens. J Thorac Cardiovasc Surg. 2007;133:1226-33.

4. Gleason TG. New graft formulation and modification of the David reimplantation technique. J Thorac Cardiovasc Surg. 2005;130:601-3.

5. Gleason TG. Aortic annuloplasty and valve-sparing root replacement: details of the primary suture line. J Thorac Cardiovasc Surg. 2006;131:502-3. 
6. Swanson M, Clark RE. Dimensions and geometric relationships of the human aortic valve as a function of pressure. Circ Res. 1974;35:871-82.

7. Ruel M, Kulik A, Lam BK, Rubens FD, Hendry PJ, Masters RG, et al. Long-term outcomes of valve replacement with modern prostheses in young adults. Eur J Cardiothorac Surg. 2005;27:425-33; discussion 33.

8. Alsoufi B, Borger MA, Armstrong S, Maganti M, David TE. Results of valve preservation and repair for bicuspid aortic valve insufficiency. $J$ Heart Valve Dis. 2005; 14:752-8; discussion 758-9.

9. Carr JA, Savage EB. Aortic valve repair for aortic insufficiency in adults: a contemporary review and comparison with replacement techniques. Eur J Cardiothorac Surg. 2004;25:6-15.

10. Casselman FP, Gillinov AM, Akhrass R, Kasirajan V, Blackstone EH, Cosgrove DM. Intermediate-term durability of bicuspid aortic valve repair for prolapsing leaflet. Eur J Cardiothorac Surg. 1999;15:302-8.

11. Gleason TG. Current perspective on aortic valve repair and valve-sparing aortic root replacement. Semin Thorac Cardiovasc Surg. 2006;18:154-64.

12. Langer F, Aicher D, Kissinger A, Wendler O, Lausberg H, Fries R, et al. Aortic valve repair using a differentiated surgical strategy. Circulation. 2004;110: II67-73.

13. Nash PJ, Vitvitsky E, Li J, Cosgrove DM III, Pettersson G, Grimm RA. Feasibility of valve repair for regurgitant bicuspid aortic valves-an echocardiographic study. Ann Thorac Surg. 2005;79:1473-9.

14. Aicher D, Kunihara T, Abou Issa O, Brittner B, Graber S, Schafers HJ. Valve configuration determines long-term results after repair of the bicuspid aortic valve. Circulation. 2011;123:178-85.
15. Asano M, Kunihara T, Aicher D, El Beyrouti H, Rodionycheva S, Schafers HJ. Mid-term results after sinotubular junction remodelling with aortic cusp repair. Eur J Cardiothorac Surg. 2012;42:1010-5.

16. Boodhwani M, de Kerchove L, Glineur D, Rubay J, Vanoverschelde JL, Van Dyck M, et al. Aortic valve repair with ascending aortic aneurysms: associated lesions and adjunctive techniques. Eur J Cardiothorac Surg. 2011;40:424-8.

17. Mosala Nezhad Z, de Kerchove L, Hechadi J, Tamer S, Boodhwani M, Poncelet A, et al. Aortic valve repair with patch in non-rheumatic disease: indication, techniques and durability. Eur J Cardiothorac Surg. 2014;doi: 10.1093/ejcts/ezu058.

18. Liu X, Weale P, Reiter G, Kino A, Dill K, Gleason T, et al. Breathhold timeresolved three-directional MR velocity mapping of aortic flow in patients after aortic valve-sparing surgery. J Magn Reson Imaging. 2009;29:569-75.

19. Sauvage LR, Wood SJ, Berger KE, Campbell AA. Autologous pericardium for mitral leaflet advancement: findings in the human after 56 months. J Thorac Cardiovasc Surg. 1966;52:849-54.

20. Frater RW, Berghuis J, Brown AL Jr, Ellis FH Jr. The experimental and clinical use of autogenous pericardium for the replacement and extension of mitral and tricuspid value cusps and chordae. J Cardiovasc Surg (Torino). 1965;6:214-28.

21. Kumar SP, Prabhakar G, Kumar M, Kumar N, Shahid M, Ali ML, et al. Comparison of fresh and glutaraldehyde-treated autologous stented pericardium as pulmonary valve replacement. J Card Surg. 1995;10:545-51.

22. David TE. Sizing and tailoring the Dacron graft for reimplantation of the aortic valve. J Thorac Cardiovasc Surg. 2005;130:243-4. 


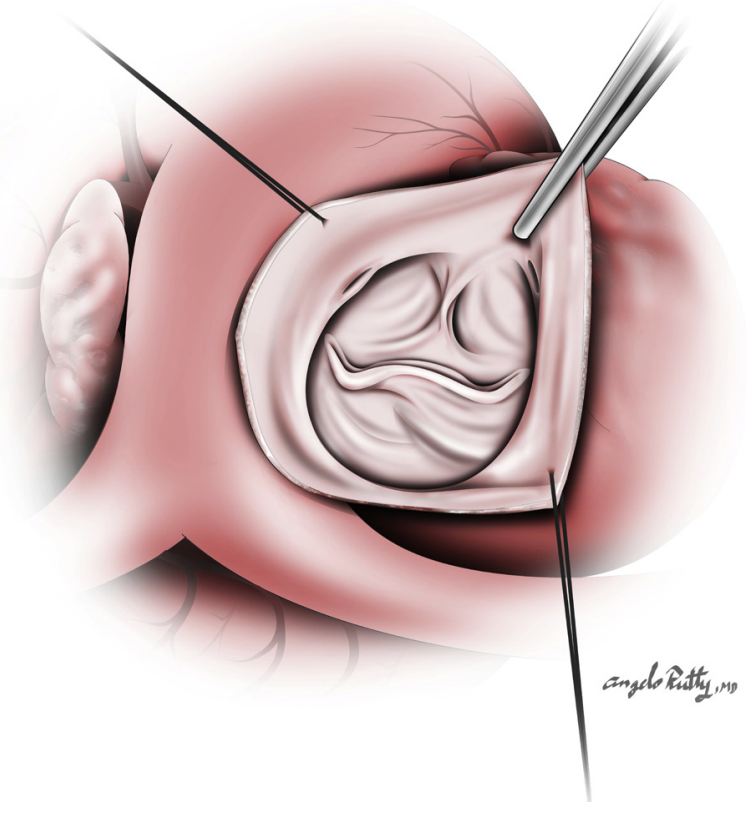

FIGURE E1. The most common phenotype of the bicuspid aortic valve (type 1) with a median raphe and false commissure oriented between the anatomic left and right coronary arteries. The posterior (noncoronary) cusp will typically be free of disease.

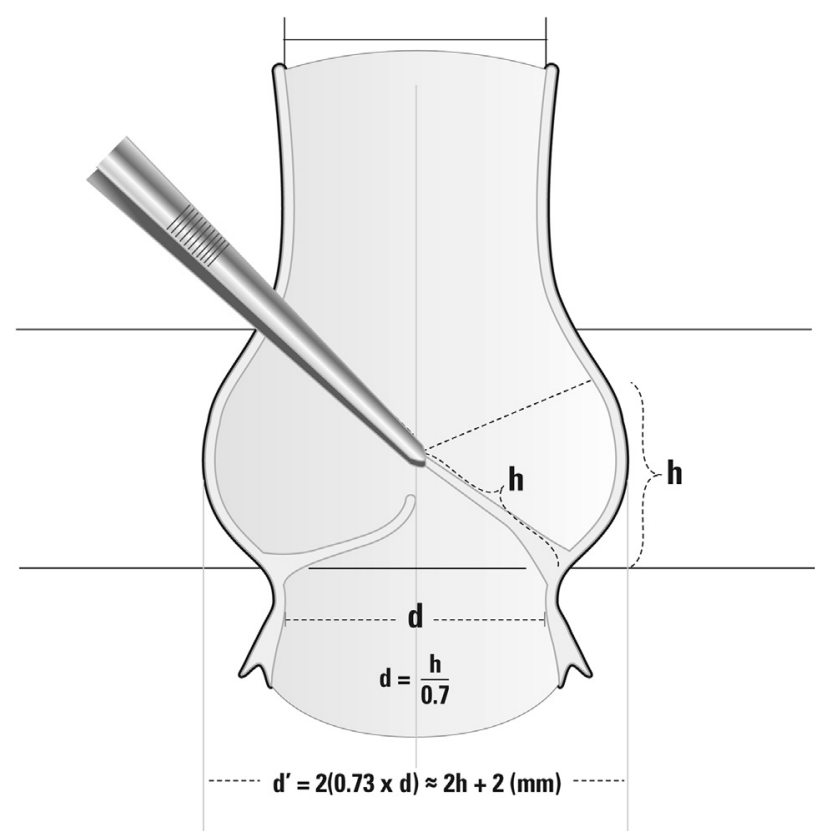

FIGURE E2. The normal geometric relationships between the cusp height $(h)$, the commissural height, the annular diameter $(d)$, and the sinus segment diameter $\left(d^{\prime}\right)$.

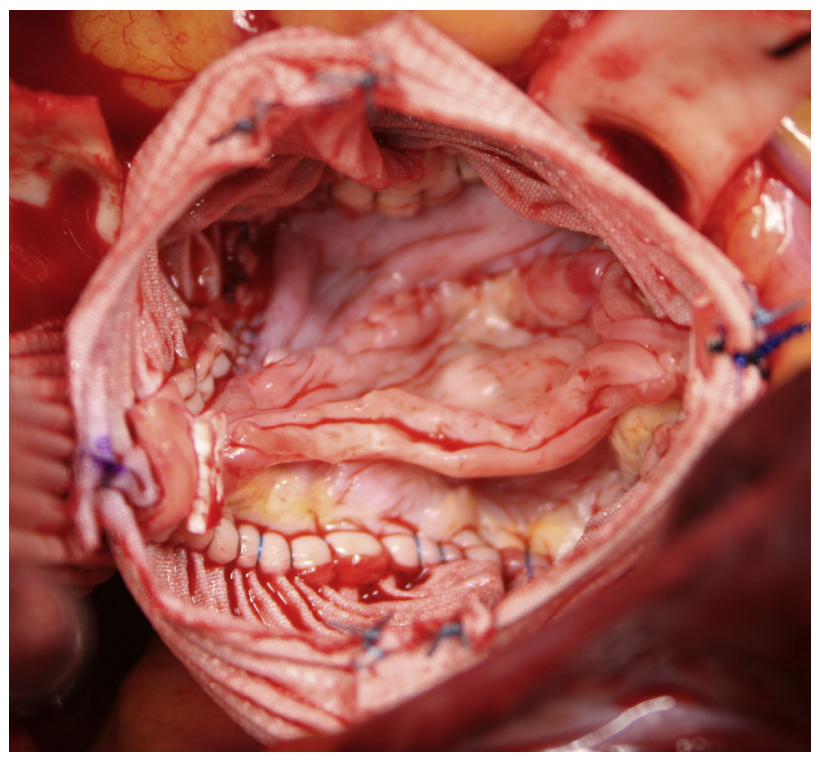

FIGURE E3. A symmetric bicuspid aortic valve with pericardial patch enlargement of its base. Note specifically that only 1 to $2 \mathrm{~mm}$ of the residual aorta was preserved, along with the native aortic annulus, with the reimplantation technique used. This allows very precise fixation of the annulus to the graft. Also note the typical position of the pleats to facilitate neosinus creation.

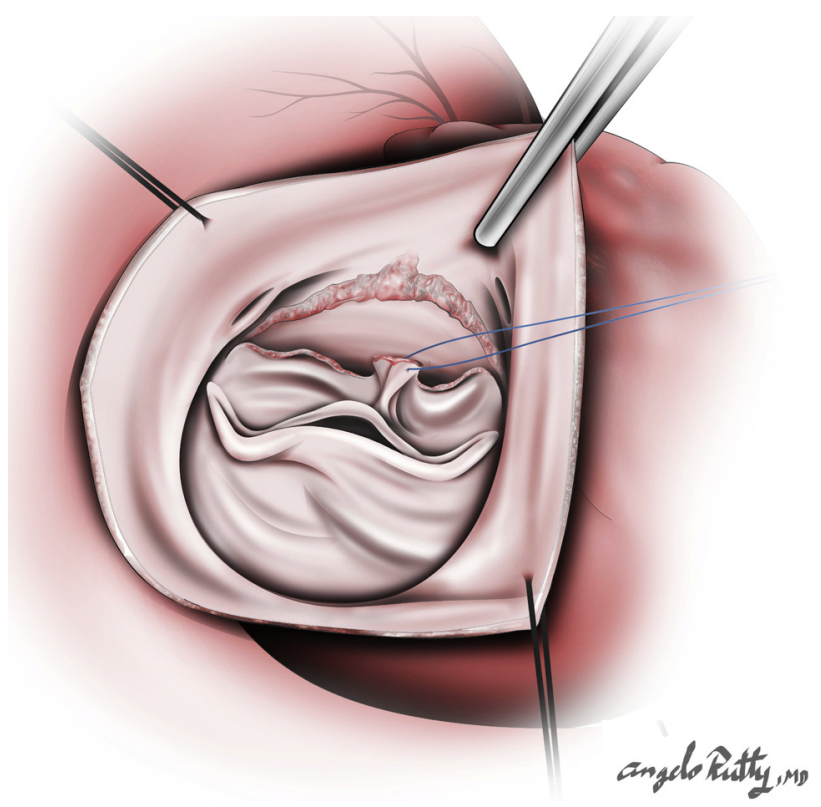

FIGURE E4. The detachment of the base of the raphe'd cusp up to the commissural attachments. 


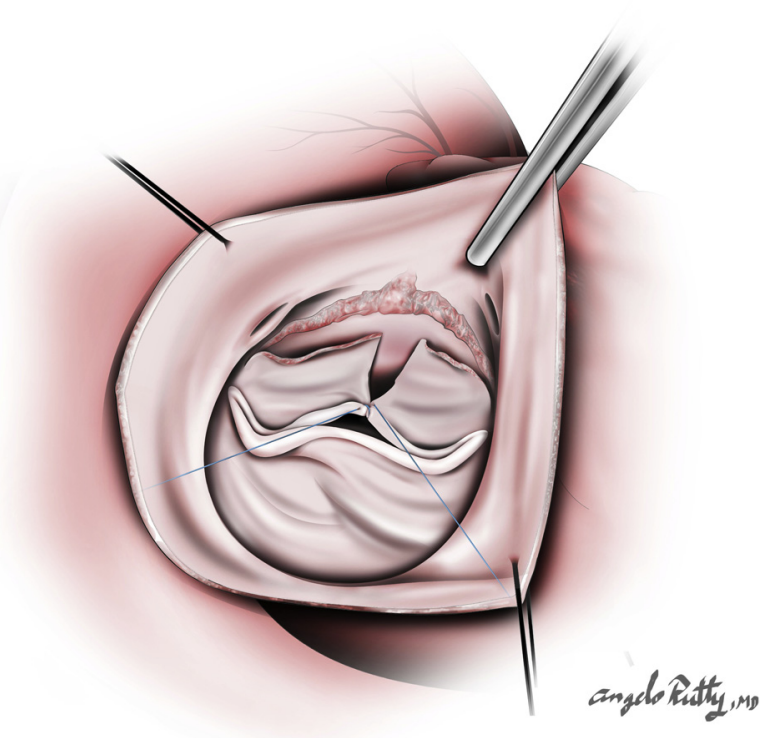

FIGURE E5. Apposition of the psreviously cut leading edges of the anterior cusp after resection of the median raphe. Note that the leading edge length of the noncoronary cusp and the tailored anterior cusp are made equivalent at this point by trimming the resection line to create 2 equal length cusps.

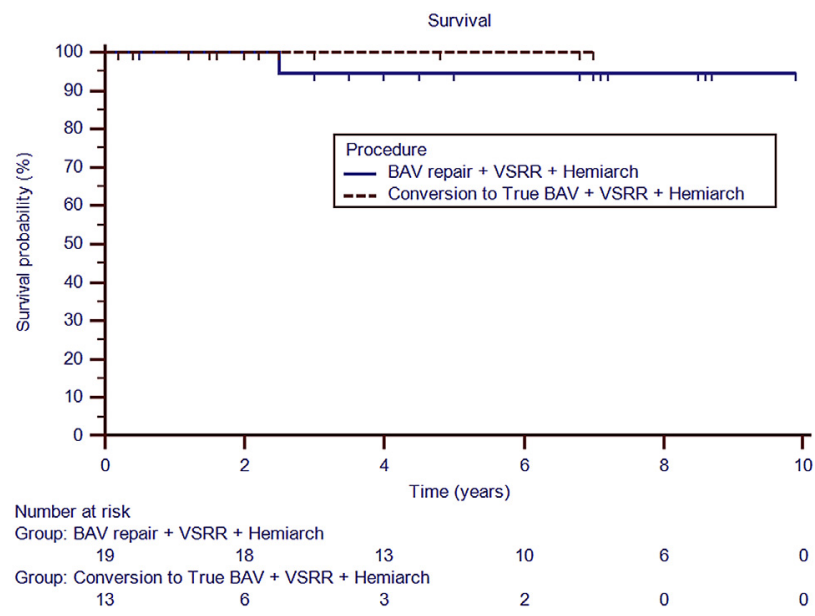

FIGURE E7. Kaplan-Meier survival curves of those who had undergone bicuspid aortic valve repair, valve-sparing root replacement, and hemiarch reconstruction (solid blue line) and those who had undergone complete conversion to a "symmetric" bicuspid valve, with valve-sparing root replacement and hemiarch reconstruction (red dashed line). BAV, Bicuspid aortic valve; VSRR, valve-sparing root replacement.

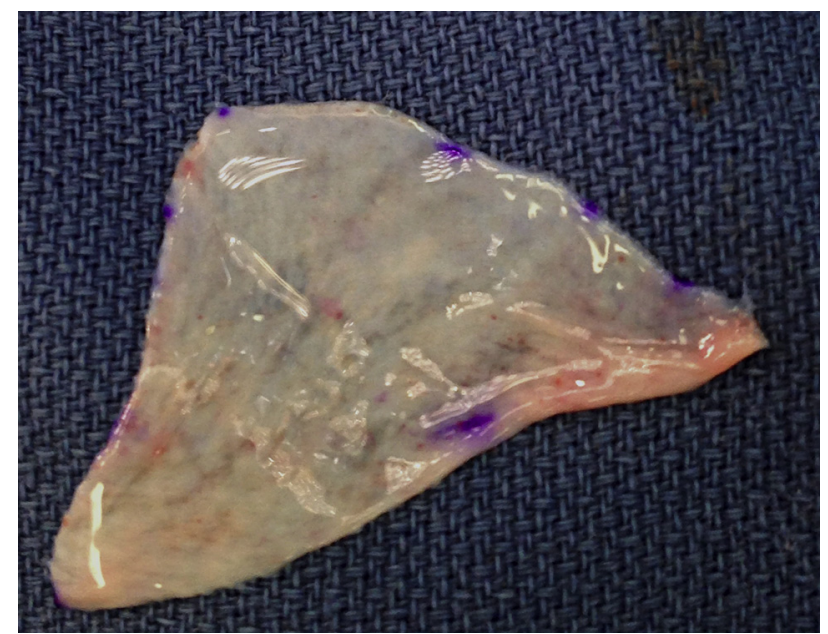

FIGURE E6. A piece of cleaned native pericardium meticulously tailored to match the size and shape of the posterior (noncoronary) cusp. 